\title{
An Improved Time-Domain Traveling-Wave Model for Vertical-Cavity Surface-Emitting Lasers
}

\author{
S. F. $\mathrm{Yu}$
}

\begin{abstract}
The use of the time-dependent transfer matrix and wide-angle beam propagation method is proposed to improve the computational speed and accuracy of a recently developed timedomain traveling-wave model of vertical-cavity surface-emitting lasers. With suitable utilization of the transfer matrix, significant reduction of the total execution time of the traveling-wave model can be obtained. In addition, the use of wide-angle beam propagation method can minimize the calculation error of the traveling-wave model due to the diffraction of light from the small aperture of the laser cavity.
\end{abstract}

Index Terms -Diffraction loss, Padé approximant propagation operator, semiconductor device modeling, surface-emitting devices, transfer matrix method.

\section{INTRODUCTION}

V ERTICAL-CAVITY surface-emitting lasers (VCSEL's) are considered to be the key components of future highspeed long-haul optical fiber communication systems because of their potential capability for stable single-mode operation, ultrahigh intrinsic relaxation oscillation frequency, ultralow threshold current condition, and efficient coupling into optical fibers. The intrinsic relaxation oscillation frequency of VCSEL's is the result of the high photon density inside the small laser cavity. It was demonstrated that the relaxation oscillation frequency of VCSEL's can be as high as $71 \mathrm{GHz}$ [1]. However, the maximum modulation bandwidth of VCSEL's is found to be limited to $14 \mathrm{GHz}$ [2]. Therefore, it is necessary to have a thorough understanding of the dynamic behavior of VCSEL's.

Simple rate-equation models are commonly used to analyze the modulation characteristics of VCSEL's [3], [4]. Nevertheless, some important characteristics of VCSEL's have been ignored in these calculations, such as: 1) the change of Bragg reflectivity due to the diffraction of light within the small cavity aperture and 2) self-focusing of the optical beam inside the active region due to the change of refractive index. In fact, these inherent properties have significant impact on: 1) the total cavity loss [5]; 2) transverse mode stability [6], [7]; and 3) modulation responses [8], [9] of VCSEL's. Hence, timedomain traveling-wave models of VCSEL's are developed with: 1) the reflectivity of the Bragg stack, 2) diffraction of light; and 3) self-focusing effects taken into consideration [8], [9]. However, the major limitations of these travelingwave models are: 1) the requirement of extensive computation

Manuscript received February 2, 1998; revised May 5, 1998. This work was supported by HK-RGC under Project HKU 7059/98E and by HKU-CRCG.

The author is with the Department of Electrical and Electronic Engineering, University of Hong Kong, Hong Kong.

Publisher Item Identifier S 0018-9197(98)07192-9. time due to the nature of the time-domain algorithm and 2) the phase errors of off-axis light diffraction due to the paraxial approximation of the propagation waves. Therefore, it is necessary to redesign the time-domain algorithm of the traveling-wave model for better computational speed and accuracy.

In this paper, the use of: 1) the time-dependent transfer matrix to improve the computational speed and 2) wideangle beam propagation method to reduce the phase errors inside each dielectric layer is proposed for the travelingwave model. In Section II, a modified time-domain travelingwave algorithm using the transfer matrix and wide-angle beam propagation method is developed. In Section III, the computational speed as well as the calculation accuracy of the time-domain traveling-wave models are examined with and without the cases of: 1) the time-dependent transfer matrix and 2) wide-angle beam propagation method taken into consideration. Finally, a brief discussion of the results and conclusions are given in Section IV.

\section{DYNAMIC MODELS}

The traveling-wave equations describing the propagation of the optical field inside the laser cavity along the longitudinal $z$ and transverse $r$ directions can be written as

$$
\begin{aligned}
\frac{1}{\nu_{g}} \frac{\partial \underline{\Psi}^{ \pm}(r, z, t)}{\partial t} \pm & \frac{\partial \underline{\Psi}^{ \pm}(r, z, t)}{\partial z} \\
& =\frac{i}{2 \beta}[H(r, z, t)] \underline{\Psi}^{ \pm}(r, z, t)
\end{aligned}
$$

where $i=\sqrt{ }-1, \nu_{g}=\left(c / n_{g}\right)$ is the group velocity, $n_{g}$ is the group refractive index, $c$ is the light velocity in free space, and $\beta$ is the longitudinal propagation coefficient. $\underline{\Psi}^{+}$and $\underline{\Psi}^{-}$ are the slowly varying envelope of the forward and reverse traveling waves, respectively, along the longitudinal direction. The effective one-dimensional (1-D) (longitudinal direction) [8] and quasi-three-dimensional (3-D) (longitudinal and transverse directions) [9] distribution of propagation fields can be calculated through $[H(r, z, t)]$ in (1). The corresponding expression of $[H(r, z, t)]$ is given by

$$
\begin{aligned}
& {[H(r, z, t)]} \\
& \quad= \begin{cases}\Delta \varepsilon_{\mathrm{eff}}(z, t) k_{o}^{2}, & \text { for 1-D } \\
\frac{1}{r} \frac{\partial}{\partial r}\left(r \frac{\partial}{\partial r}\right)^{2}+\Delta \varepsilon(r, z, t) k_{o}^{2}, & \text { for quasi 3-D }\end{cases}
\end{aligned}
$$

where $k_{o}=\left(2 \pi / \lambda_{o}\right)$ is the wavevector, $\lambda_{o}$ is the wavelength, $\Delta \varepsilon_{\text {eff }}$ and $\Delta \varepsilon$ are the effective change of permittivity of 
1-D and quasi-3-D waveguide geometry, respectively (see Appendix A). Therefore, (1) can be used to describe 1-D or quasi-3-D distributions of the optical field inside the laser cavity depending on the use of the operator $[\mathrm{H}]$.

Equation (1) can be solved by using the time-domain traveling-wave method as given in [10]. The time and spatial variation of wave equations can be linearized by a first-order difference approximation to the partial differential in (1). By choosing the relation between time and the spatial step as $\Delta z=\nu_{g} \cdot \Delta t$, we have

$$
\begin{aligned}
& \Psi^{ \pm}(r, z \pm \Delta z, t+\Delta t) \\
& \quad=[1+i[H(r, z, t)] \cdot \Delta z / 2 \beta] \Psi^{ \pm}(r, z, t)
\end{aligned}
$$

where $\Delta t$ is a constant time step and $\Delta z$ is the propagation distance. If the initial value of $\Psi^{ \pm}(r, z, t)$ at time $t$ and location $z$ is known, the fields $\Psi^{ \pm}(r, z, t+\Delta t)$ at the next time step $t+\Delta t$ can be determined at the position $z \pm \Delta z$ from (3). In addition, the boundary conditions at the interface of the dielectric layers are applied to $\Psi^{ \pm}(r, z \pm \Delta z, t+\Delta t)$ for the calculation of the reflectivity of the alternate dielectric layers such that the reflectivity of the Bragg reflectors is evaluated simultaneously. As we can see, the time-domain algorithm has the advantages of easy implementation and implicit introduction of longitudinal modes into the calculation. However, the total execution time of the time-domain traveling-wave model of VCSEL's is dependent on its propagation distance $\Delta z$ and the total number of dielectric layers. This is because the decrease in $\Delta z$ leads to an increase in the number of computational steps due to the requirement of time and the spatial step relation (i.e., $z=\nu_{g} \cdot t$ ). Furthermore, the Bragg reflectivities are calculated by applying boundary conditions at the interface of the dielectric layers such that the value of $\Delta z$ used in (3) should equal the physical thickness of the dielectric layers [8], [9]. It can be shown that the value of $\Delta z$ is about $0.1 \mu \mathrm{m}$ (using $\lambda_{o}=1.55 \mu \mathrm{m}$ and $n_{g}=3.7$ ) which is shorter than that used in distributed feedback (DFB) lasers (i.e., $2 \mu \mathrm{m}$ ) [10]. Therefore, the disadvantages of using the time-domain algorithm for VCSEL's are: 1) the number of computational steps depends on the total number of dielectric layers; 2) $\Delta z$ depends on the thickness of the dielectric layers; and 3) $\Delta z$ is too small. In addition, the execution time for the quasi-3D model will be much longer than that of the 1-D model of VCSEL's due to the existence of the transverse dimension.

\section{A. Time-Domain Traveling-Wave Model Using the Transfer Matrix}

In order to solve the problem of small $\Delta z$ but without sacrificing the advantages of the time-domain algorithm, we modify the above model of VCSEL's by using the transfer matrix method [11]. Fig. 1 illustrates the use of a transfer matrix $[S(r, z, t)]_{1}$ to represent a dielectric layer. The propagation fields can be related to $[S(r, z, t)]_{1}$ by

$$
\begin{aligned}
& {\left[\begin{array}{c}
\underline{\Psi}^{+}(r, z+\Delta z, t+\Delta t) \\
\underline{\Psi}^{-}(r, z+\Delta z, t)
\end{array}\right]} \\
& \quad=\left[\begin{array}{ll}
s_{11}(r, z, t) & s_{12}(r, z, t) \\
s_{21}(r, z, t) & s_{22}(r, z, t)
\end{array}\right]_{1}\left[\begin{array}{c}
\underline{\Psi}^{+}(r, z, t) \\
\underline{\Psi}^{-(r, z, t+\Delta t)}
\end{array}\right]
\end{aligned}
$$
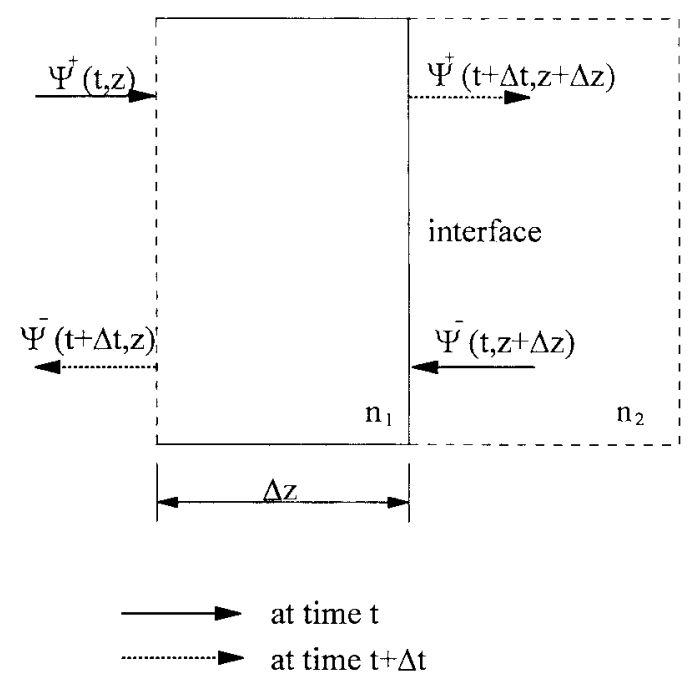

Fig. 1. Schematic illustrating the use of the transfer matrix in the time-domain algorithm.

where the subscript 1 of $[S(r, z, t)]$ represents the position of the dielectric layer, $s_{h, k}$ are the elements of the transfer matrix $[S(r, z, t)]_{1}$, and $h, k=1,2$. The transfer matrix can be determined by considering the propagation of traveling waves along the dielectric layer and the transmission and reflection between the interface of the adjacent dielectric layers.

The propagation of forward and reverse propagating waves $\underline{\Psi}^{+}$and $\underline{\Psi}^{-}$along the dielectric layer can be expressed as

$$
\left[\begin{array}{l}
\underline{\Psi}^{+} \\
\underline{\Psi}^{-}
\end{array}\right]_{r, z+\Delta z, t}=\left[\begin{array}{cc}
\exp ([H] \cdot \Delta z) & 0 \\
0 & \exp (-[H] \cdot \Delta z)
\end{array}\right]\left[\underline{\underline{\Psi}}^{+}\right]_{r, z, t} .
$$

In addition, the transfer matrix for a refractive index step from a dielectric layer of refractive index $n_{1}$ to that of refractive index $n_{2}$ is given by

$$
\left[\begin{array}{l}
\underline{\Psi}^{+} \\
\underline{\Psi}^{-}
\end{array}\right]_{\text {at } n_{2}}=\left[T_{21}\right]^{-1}\left[\begin{array}{cc}
1 & {\left[R_{21}\right]} \\
{\left[R_{21}\right]} & 1
\end{array}\right]\left[\begin{array}{l}
\underline{\Psi}^{+} \\
\underline{\Psi}^{-}
\end{array}\right]_{\text {at } n_{1}}
$$

where $\left[R_{21}\right]$ and $\left[T_{21}\right]$ are the reflection and transmission coefficients, respectively, and their corresponding expressions are given in the Appendix B. It must be noted the influence of off-axis reflection and transmission at the dielectric interfaces has been taken into consideration through the proper assessment of $\left[R_{21}\right]$ and $\left[T_{21}\right]$ in (6). Therefore, the transfer matrix $[S(r, z, t)]_{1}$ can be written as

$$
\begin{aligned}
{\left[\begin{array}{cc}
s_{11}(r, z, t) & s_{12}(r, z, t) \\
s_{21}(r, z, t) & s_{22}(r, z, t)
\end{array}\right]_{1} } \\
=\left[T_{21}\right]^{-1}\left[\begin{array}{cc}
1 & {\left[R_{21}\right]} \\
{\left[R_{21}\right]} & 1
\end{array}\right] \\
\cdot\left[\begin{array}{cc}
\exp ([H] \cdot \Delta z) & 0 \\
0 & \exp (-[H] \cdot \Delta z)
\end{array}\right] .
\end{aligned}
$$

The exponential terms in (7) can be simplified to improve the computing efficiency as

$$
\begin{aligned}
\exp ( \pm[H] \cdot \Delta z)= & {[I] \pm[H] \cdot \Delta z+\frac{1}{2}[H]^{2} \cdot \Delta z^{2} \pm \frac{1}{6}[H]^{3} } \\
& \cdot \Delta z^{3}+\frac{1}{24}[H]^{4} \cdot \Delta z^{4} \pm \cdots
\end{aligned}
$$


where $[I]$ is an identity matrix. The time-domain algorithm given in [10] can be utilized to solve (4). This can be done by rewriting (4) in the following format:

$$
\begin{aligned}
& {\left[\begin{array}{c}
\underline{\Psi}^{+}(r, z+\Delta z, t+\Delta t) \\
\underline{\Psi}^{-}(r, z, t+\Delta t)
\end{array}\right]} \\
& =s_{22}^{-1}\left[\begin{array}{cc}
\left(s_{11} \cdot s_{22}-s_{12} \cdot s_{21}\right) & s_{12} \\
1 & -s_{21}
\end{array}\right] \\
& \cdot\left[\begin{array}{c}
\underline{\Psi}^{+}(r, z, t) \\
\underline{\Psi}^{-(r, z+\Delta z, t)}
\end{array}\right] .
\end{aligned}
$$

Now, (9) can be solved by advancing $\Psi^{ \pm}(r, z, t)$ from one dielectric layer to another during each time interval $\Delta t$. In addition, the boundary conditions between dielectric layers are implicitly introduced into (9) such that the reflectivity of Bragg reflectors can also be evaluated in a time-dependent manner. The main differences of this model to that without the transfer matrix are: 1) the boundary conditions are implicitly introduced into consideration and 2) the propagating distance of $\Psi^{ \pm}(r, z, t)$ is not restricted to $\Delta z$ as in [8], [9] but is varied with the propagation length of the transfer matrix.

Now, the total number of computational steps as well as the execution time of the traveling-wave model can be reduced by increasing the propagation length of the transfer matrix. For example, if a new transfer matrix is defined as $[S(r, z, t)]_{1} \times[S(r, z, t)]_{2}$, the corresponding propagation distance of this transfer matrix is increased to $2 \Delta z$ such that the number of computational steps as well as the execution time can be reduced roughly by a factor of two.

\section{B. Time-Domain Traveling-Wave Algorithm Using Wide-Angle Beam Propagation Method}

For VCSEL's with diffraction of light occurring inside the laser cavity, especially for devices with small aperture such as the index-guided devices [12], the amplitude and phase variation of the traveling wave are nonuniformly distributed over the transverse direction within each dielectric layer. Therefore, the assumption of a uniform plane wave is not valid and the term $\partial^{2} / \partial z^{2}$ should be included in the travelingwave model such that the superposed waves traveling at widely different off-axis angles (i.e., wide-angle propagation) can be calculated. The corresponding wave equation can be written as

$$
\begin{aligned}
\frac{1}{\nu_{g}} & \frac{\Psi^{ \pm}(r, z, t)}{\partial t} \pm \frac{\partial \underline{\Psi}^{ \pm}(r, z, t)}{\partial z} \\
& =\frac{i}{2 \beta}\left[\frac{1}{r} \frac{\partial}{\partial r}\left(r \frac{\partial}{\partial r}\right)+\frac{\partial^{2}}{\partial z^{2}}+\Delta \varepsilon(r, z, t) k_{o}^{2}\right] \underline{\Psi}^{ \pm}(r, z, t)
\end{aligned}
$$

where $\partial^{2} / \partial z^{2}$ is included into the wave equations to take into account the influence of diffraction.

The displacement and time variation of $\underline{\Psi}^{ \pm}(r, z, t)$ given in (10) can be solved by using the Padé recursion approximation [13] and the time-dependent transfer matrix [11], respectively. First, rewrite (10) in a matrix format to describe the propagation of $\underline{\Psi}^{ \pm}$for a distance $\Delta z$ from $z$ and at a time $t$ as in (5).
The traveling-wave equation of $\underline{\Psi}^{+}$can be expressed as

$$
\begin{aligned}
& \frac{\partial}{\partial z}\left[1-\frac{i}{2 \beta} \frac{\partial}{\partial z}\right] \underline{\Psi}^{+}(r, z) \\
& \quad=\frac{i}{2 \beta}\left[\frac{1}{r} \frac{\partial}{\partial r}\left(r \frac{\partial}{\partial r}\right)+\Delta \varepsilon(r, z) d_{o}^{2}\right] \underline{\Psi}^{+}(r, z)
\end{aligned}
$$

where the time derivative term $\partial / \partial t$ in (10) has been ignored in the derivation. Equating the operators on $\underline{\Psi}^{+}$on the leftand right-hand sides of (11), we obtain the Padé recursion approximation on (11) as

$$
\frac{\partial}{\partial z}=\frac{\frac{i}{2 \beta}[H(r, z, t)]}{1-\frac{i}{2 \beta} \frac{\partial}{\partial z}} \equiv[P]
$$

where $[P]$ treats the field variation along the transverse direction. Therefore, $\underline{\Psi}^{+}$at distance $z+\Delta z$ can be obtained by integrating (12) along the longitudinal direction, and the solution is given by

$$
\underline{\Psi}^{+}(r, z+\Delta z)=\exp ([P] \cdot \Delta z) \underline{\Psi}^{+}(r, z) .
$$

The expression of $\underline{\Psi}^{-}$can also be determined in a similar approach. Therefore, the time-independent transfer matrix of (10) can be expressed as

$$
\begin{aligned}
& {\left[\begin{array}{l}
\underline{\Psi}^{+} \\
\underline{\Psi}^{-}
\end{array}\right]_{r, z+\Delta z, t}} \\
& \quad=\left[\begin{array}{cc}
\exp ([P] \cdot \Delta z) & 0 \\
0 & \exp (-[P] \cdot \Delta z)
\end{array}\right]\left[\begin{array}{l}
\underline{\Psi}^{+} \\
\underline{\Psi}^{-}
\end{array}\right]_{r, z, t} .
\end{aligned}
$$

Second, let us take $\Delta z=\Delta t \cdot \nu_{g}$. Then the time-dependent transfer matrix of (10) can be written as in (7), that is

$$
\begin{aligned}
{\left[\begin{array}{cc}
s_{11}(r, z, t) & s_{12}(r, z, t) \\
s_{21}(r, z, t) & s_{22}(r, z, t)
\end{array}\right]_{1} } \\
=\left[T_{21}\right]^{-1}\left[\begin{array}{cc}
1 & {\left[R_{21}\right]} \\
{\left[R_{21}\right]} & 1
\end{array}\right] \\
\cdot\left[\begin{array}{cc}
\exp ([P] \cdot \Delta z) & 0 \\
0 & \exp (-[P] \cdot \Delta z)
\end{array}\right]
\end{aligned}
$$

where the exponential terms in (15) can be simplified as

$$
\begin{aligned}
\exp ( \pm[P] \cdot \Delta z)= & {[I] \pm[P] \cdot \Delta z+\frac{1}{2}[P]^{2} \cdot \Delta z^{2} \pm \frac{1}{6}[P]^{3} } \\
& \cdot \Delta z^{3}+\frac{1}{24}[P]^{4} \cdot \Delta z^{4} \pm \cdots
\end{aligned}
$$

By substituting (15) into (9), the displacement and time variation of $\underline{\Psi}^{ \pm}$can be solved using the time-domain algorithm. It must be noted that the off-axis propagation of light at the interface of the dielectric layers has also been properly taken into account through $\left[R_{21}\right]$ and $\left[T_{21}\right]$. Hence, a very simple but powerful quasi-3-D dynamic model for VCSEL's is developed with full consideration of light diffraction inside the laser cavity.

The calculation of the phase of the propagation fields using (12) can be simplified by the Padé recursion approximation on 
$[P]$. This is because (12) suggests a recurrence relation

$$
\left.\frac{\partial}{\partial z}\right|_{j}=\frac{\frac{i}{2 \beta}[H(r, z, t)]}{1-\left.\frac{i}{2 \beta} \frac{\partial}{\partial z}\right|_{j-1}}
$$

where $j$ is an integer. The low-order Padé operators that result from the application of (17) can be obtained by the recurrence relation. For example, the Padé $(1,1),(2,2)$, and $(3,3)$ operators can be written as seen in (18)-(20), shown at the bottom of the page.

In fact, higher-order Padé operators can also be obtained in the same manner. It has been discussed in [13] that phase error can be minimized by the Padé $(1,1)$ operator or almost suppressed by the Padé $(3,3)$ operator, even at a propagation angle of $30^{\circ}$. Therefore, a Padé operator up to the order of (3, 3 ) is sufficient for use in the traveling-wave model of VCSEL for the consideration of light diffraction.

\section{Electrical Model Using the Rate Equation for Carrier Concentration}

The electrical part of the dynamic model of VCSEL's used in this analysis is similar to that given in [9]. The nonuniform distribution of carrier concentration $N(r, t)$ inside the active layer is described by the carrier rate equation as shown below:

$$
\begin{aligned}
\frac{\partial N(r, t)}{\partial t}= & \frac{J(r, t)}{e d}-R(r, t)-\nu_{g} g(N) E(r, t) \\
& +D_{f} \frac{1}{r} \frac{\partial}{\partial r}\left(r \frac{\partial N(r, t)}{\partial r}\right)
\end{aligned}
$$

where $e$ is the electron charge, $d$ is total thickness of wells, $g(N)\left(=a_{N} \cdot \log \left(N / N_{o}\right)\right)$ is the optical gain, $a_{N}$ is a fitted parameter, and $N_{o}$ is the carrier concentration at transparency. $E\left(=\left|\Psi^{+}\right|^{2}+\left|\Psi^{-}\right|^{2}\right)$ is the photon density inside the active layer and $D_{f}$ is the diffusion coefficient. $R(r, t)$ is the recombination rate of carrier concentration and is defined as

$$
R(r, t)=N(r, t) / \tau+B N(r, t)^{2}+C N(r, t)^{3}
$$

where $\tau$ is the carrier lifetime, $B$ is the bimolecular recombination coefficient, and $C$ is the Auger recombination coefficient of carrier. $J(r, t)$ is the current density distribution along the transverse direction and is given by [14]

$$
J(r, t)= \begin{cases}J_{o}, & r \leq w \\ J_{o} \exp \left(-(r-w) / r_{o}\right), & r>w\end{cases}
$$

where $J_{O}$ denotes the current density at the edge and within the contact area $(r \leq w)$, and $r_{o}$ is the effective diffusion length of the injection carrier.

The transient response of VCSEL's can be calculated by solving the wave equations and the rate equation of carrier concentration by a time-domain algorithm in a self-consistent manner [8]. This can be done by relating the time and spatial steps in the longitudinal direction as $\Delta t=\Delta z / \nu_{g}$. Therefore, at each propagation distance of the traveling waves, the corresponding change of carrier concentration can be written as

$$
\begin{gathered}
N(r, t+\Delta t)-N(r, t) \\
=\frac{1}{\nu_{g}}\left(\frac{J(r, t)}{e d}-R(r, t)-\nu_{g} g(N) E(r, t)\right. \\
\left.\quad+D_{f} \frac{1}{r} \frac{\partial}{\partial r}\left(r \frac{\partial N(r, t)}{\partial r}\right)\right) \Delta z .
\end{gathered}
$$

It must be noted that (24) is computed during each computational step of the traveling-wave equations. Therefore, the execution time of (24) can be reduced by increasing the propagation distance $\Delta z$ of the time-domain algorithm. For example, if the propagation distance of the time-domain algorithm is increased from $\Delta z$ to $2 \Delta z$, the corresponding execution time of (24) will be reduced by half. Therefore, it is expected that the total execution time for the traveling-wave equations will be reduced by more than a factor of two.

\section{NUMERICAL ANALYSIS}

\section{A. Device Structure}

A schematic of the VCSEL's used in the calculation is shown in Fig. 2. The device has a built-in index-guided structure, and a circular metal contact is formed on the epitaxial side (p-side) for current injection. A InGaAsP-InP quantum-well active layer is sandwiched between two undoped spacer layers and two Bragg reflectors. The Bragg reflectors

$$
\begin{aligned}
{[P]_{(1,1)}=} & \frac{\frac{i}{2 \beta}[H(r, z, t)]}{1+\frac{1}{4 \beta^{2}}[H(r, z, t)]} \\
{[P]_{(2,2)}=} & \frac{\frac{i}{2 \beta}[H(r, z, t)]+\frac{i}{4 \beta^{3}}[H(r, z, t)]^{2}}{1+\frac{3}{4 \beta^{2}}[H(r, z, t)]+\frac{1}{16 \beta^{4}}[H(r, z, t)]^{2}} \\
{[P]_{(3,3)}=} & \frac{\frac{i}{2 \beta}[H(r, z, t)]+\frac{i}{3 \beta^{3}}[H(r, z, t)]^{2}+\frac{3 i}{32 \beta^{5}}[H(r, z, t)]^{3}}{1+\frac{5}{4 \beta^{2}}[H(r, z, t)]+\frac{3}{8 \beta^{4}}[H(r, z, t)]^{2}+\frac{1}{64 \beta^{6}}[H(r, z, t)]^{3}}
\end{aligned}
$$


TABLE I

REFRACTIVE INDEX Distribution IN THE LASER

\begin{tabular}{c|c|c|c}
\hline Refractive index of layerstregions & Core region & $\begin{array}{c}\text { Cladding } \\
\text { region }\end{array}$ & $\begin{array}{c}\text { Effective index of } \\
\text { each layer (ne) }\end{array}$ \\
\hline Active layer & 3.525 & 3.475 & 3.521 \\
\hline Spacer layer & 3.396 & 3.346 & 3.392 \\
\hline High index layer of Bragg reflector & 3.508 & 3.458 & 3.504 \\
\hline Low index layer of Bragg reflector & 2.956 & 2.906 & 2.952 \\
\hline
\end{tabular}

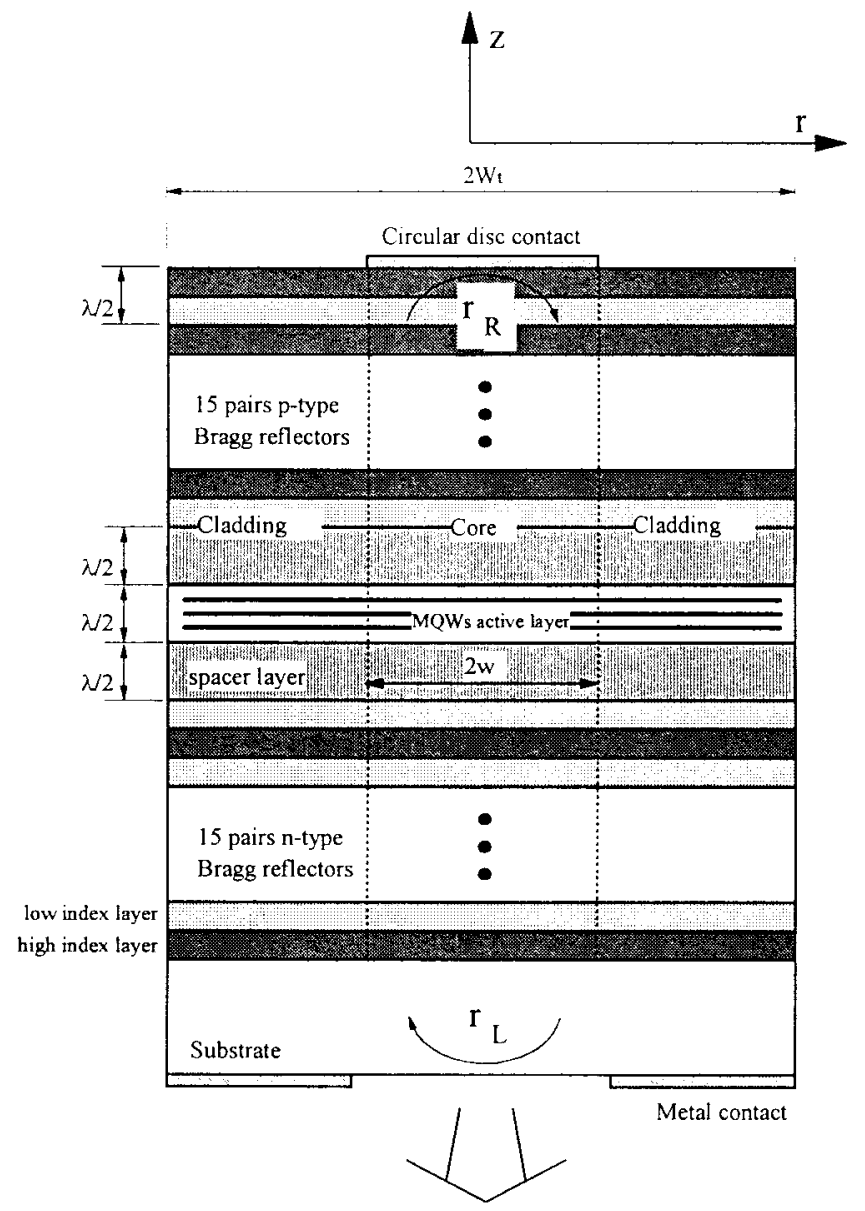

Output Power

Fig. 2. Schematic of an index-guided VCSEL.

are formed by alternating layers of GaAs and AlGaAs and consist of 13 such pairs on both the $\mathrm{n}$ - and p-sides. The radius of the core region is set to $0.8 \mu \mathrm{m}$. The corresponding values of refractive index along the longitudinal direction of the entire multilayer are listed in Table I. In addition, the material parameters used in the electrical model can be found in Table II.

\section{B. Computational Speed of Time-Domain Traveling-Wave Models Using the Transfer Matrix}

Using the laser structure given in Section III-A, the computational speed of 1-D and quasi-3-D time-domain travelingwave models is investigated. This can be done by examining the dynamic response of VCSEL's under large-signal modulation. It is assumed that the laser is biased at threshold and modulated with a step current. The modulation amplitude is

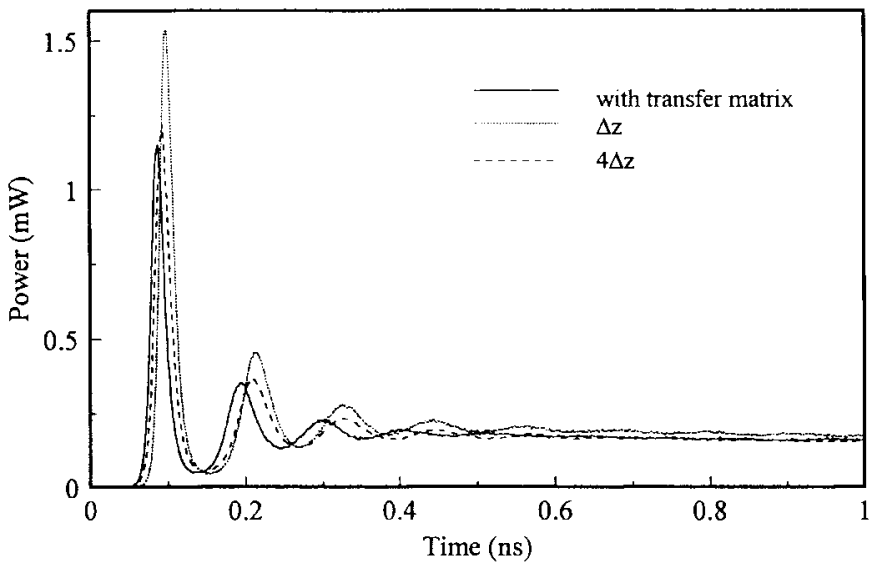

(a)

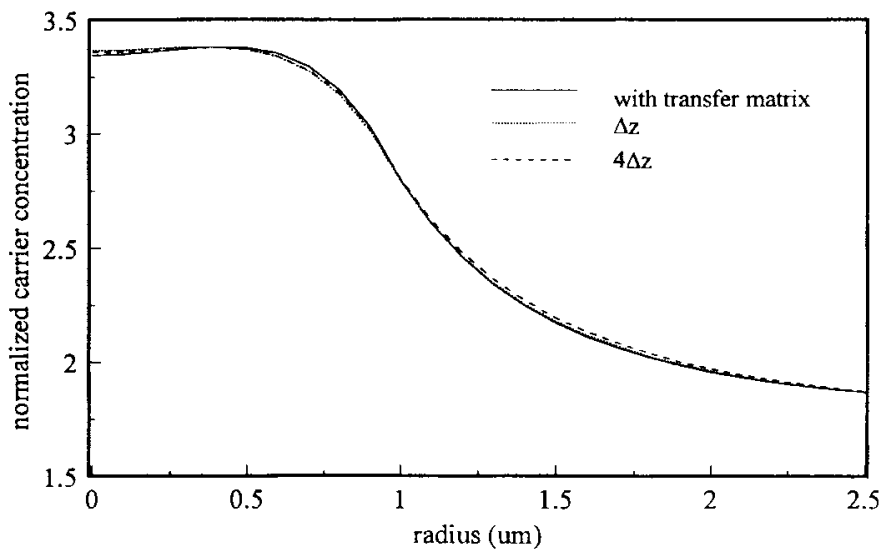

(b)

Fig. 3. The influence of transfer matrices on (a) the transient response of output power and (b) the steady-state carrier concentration distribution of a 1-D time-domain traveling-wave model.

selected such that the steady-state output power is around 0.2 $\mathrm{mW}$. The output power $P$ is defined as

$$
\boldsymbol{P}=\nu_{g} h v\left(1-\left|r_{L}\right|^{2}\right) \int_{0}^{w} \int_{0}^{2 \pi}\left|\Psi^{+}(z=0, r)\right|^{2} r d r d \theta
$$

where $h$ is Planck's constant, $r_{L}$ is the reflectivity between the air and substrate, $w$ is the core radius, and $\nu$ is the operation frequency of the laser.

Fig. 3 shows the dynamic response of the output power and the steady-state distribution of carrier concentration calculated by the 1-D time-domain traveling-wave models with different propagation distances (i.e., $\Delta z, 2 \Delta z$, and $4 \Delta z$ where $\Delta z=$ $\lambda_{o} / 4 / n e_{j}$ and $n e_{j}$ is the effective refractive index of the $j$ th dielectric layer) of the transfer matrices. Furthermore, the dynamic response of the output power and the steady-state carrier concentration distribution calculated by the quasi-3-D time- 
TABLE II

PARAMETERS USED IN THE MODEL

\begin{tabular}{l|l|l}
\hline Parameter & Symbol & Valuc \\
\hline \hline lasing wavelength & $\lambda_{\mathrm{o}}$ & $1.55 \mu \mathrm{m}$ \\
\hline group refractive index & $\mathrm{n}_{\mathrm{g}}$ & 3.7 \\
\hline carrier lifetime & $\tau$ & $3 \times 10^{-9} \mathrm{~s}$ \\
\hline Bimolecular carrier recombination coefficient & $\mathrm{B}$ & $1 \times 10^{-10} \mathrm{~cm}^{6} \mathrm{~s}^{-1}$ \\
\hline Auger carrier recombination coefficient & $\mathrm{C}$ & $3 \times 10^{-29} \mathrm{~cm}^{6} \mathrm{~s}^{-1}$ \\
\hline active layer thickness & $\mathrm{d}$ & $0.02 \mu \mathrm{m}$ \\
\hline diffusion coefficient of carrier & $\mathrm{D}_{\mathrm{f}}$ & $10 \mathrm{~cm}^{2} \mathrm{~s}^{-1}$ \\
\hline effective diffusion length of injection carrier & $\mathrm{r}_{\mathrm{O}}$ & $0.02 \mu \mathrm{m}$ \\
\hline gain parameter & $\mathrm{a}_{\mathrm{N}}$ & $1500 \mathrm{~cm}^{-1}$ \\
\hline transparency carrier concentration & $\mathrm{N}_{\mathrm{o}}$ & $2.0 \times 10^{18} \mathrm{~cm}^{-3}$ \\
\hline surface reflectivity (near substrate) & $\mathrm{r}_{\mathrm{L}}$ & 0.55 \\
\hline surface reflectivity (near circular metal contact) & $\mathrm{r}_{\mathrm{R}}$ & 0.974 \\
\hline scattering \& absorption loss in waveguide & $\alpha_{\mathrm{S}}$ & $10 \mathrm{~cm}^{-1}$ \\
\hline refractive index coefficient & $\mathrm{b}_{\mathrm{N}}$ & -0.0234 \\
\hline normalization parameter & $\mathrm{N}_{\mathrm{l}}$ & $2.45 \times 10^{18} \mathrm{~cm}^{-3}$ \\
\hline
\end{tabular}

domain traveling-wave models are shown in Fig. 4(a) and (b). The number of grid points (i.e., along the transverse direction) is set to 25 in the calculation. It is observed that the propagation distances of the transfer matrix have less influence on the 1-D and quasi-3-D time-domain calculation of the dynamic response of output power. From the above analysis, we can conclude that the introduction of the transfer matrix into the time-domain algorithm has only negligible detrimental effects on the computational accuracy of the traveling-wave models.

Fig. 5(a) and (b) shows the total execution time of the 1-D and the quasi-3-D time-domain traveling-wave models against the propagation distance of the transfer matrices. The simulation program is written in FORTRAN 90 run on an IBM Pentium II $200 \mathrm{MHz}$ PC. It is shown in Fig. 5(a) that the total execution time for the 1-D model is reduced by more than $2.5 \times$ when the propagation distance is increased from $\Delta z$ to $2 \Delta z$. This is expected because the total computational steps of (9) and (24) are reduced by half through the increase of propagation distance. Hence, the total execution time for the traveling model is reduced by more than half. However, the total execution time for the quasi-3-D model is increased by the application of the transfer matrix for a propagation distance less than $4 \Delta z$ [see Fig. 5(b)]. This is because the inverse operation of a matrix [involved in the calculation of (9)] consumes half of the total execution time of the entire model. On the other hand, the 1-D model requires the multiplication and addition of complex numbers in the calculation of (9) such that a significant improvement in total computational speed can be obtained.

\section{Minimization of Calculation Errors Due to Light Diffraction Using the Wide-Angle Beam Propagation Method in the Time-Domain Traveling-Wave Models}

Using the laser structure given in Section III-A, the calculation results obtained from the models using a paraxial propagator [i.e., without $\partial^{2} / \partial z^{2}$ in (10)] and $(3,3)$ Padé

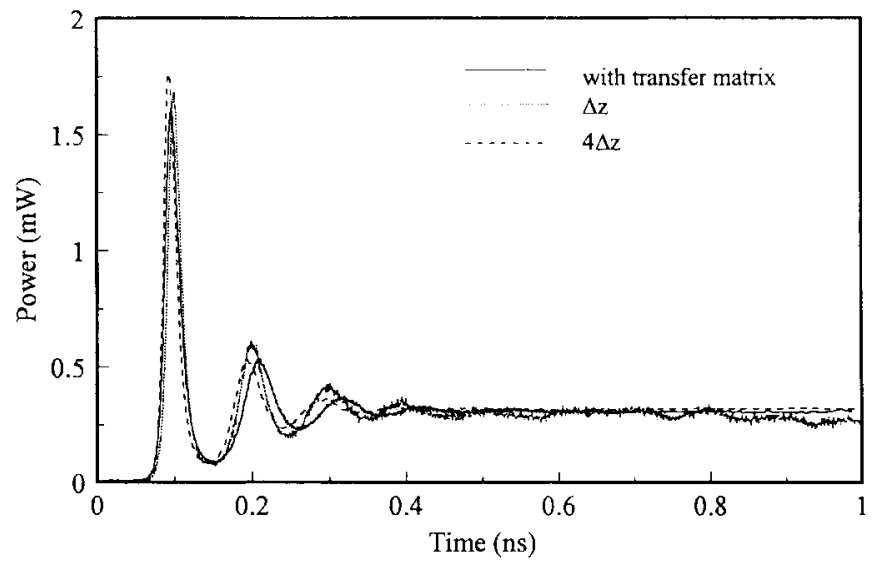

(a)

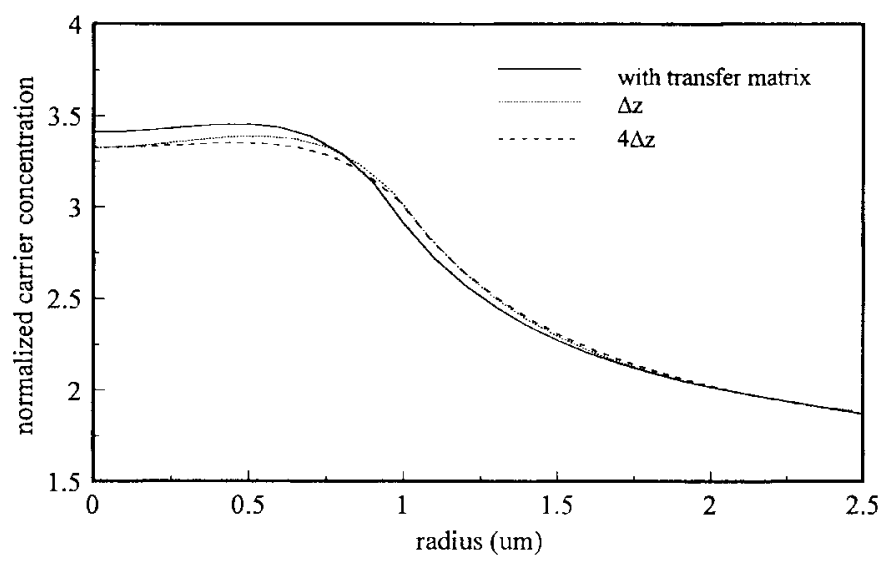

(b)

Fig. 4. The influence of transfer matrices on (a) the transient response of output power and (b) the steady-state carrier concentration distribution of a quasi-3-D time-domain traveling-wave model.

approximation operator are compared. Fig. 6(a) and (b) shows the transient response of the output power and the carrier concentration profile at steady state calculated from the models. As 
TABLE III

REFRACTIVE INDEX DisTRIBUTION IN THE LASER

\begin{tabular}{c|c|c|c}
\hline Refractive index of layers\regions & Core region & $\begin{array}{c}\text { Cladding } \\
\text { region }\end{array}$ & $\begin{array}{c}\text { Effective index of } \\
\text { each layer (ne) }\end{array}$ \\
\hline \hline Active layer & $3.6-3.4$ & 3.0 & $3.554-3.369$ \\
\hline Spacer layer & 3.392 & 3.392 & 3.392 \\
\hline High index layer of Bragg reflector & 3.504 & 3.504 & 3.504 \\
\hline Low index layer of Bragg reflector & 2.952 & 2.952 & 2.952 \\
\hline
\end{tabular}

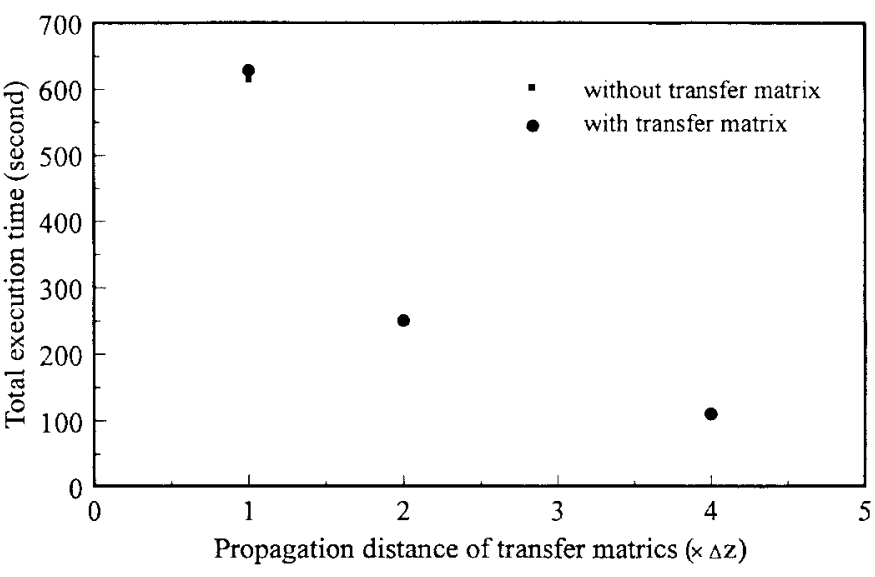

(a)

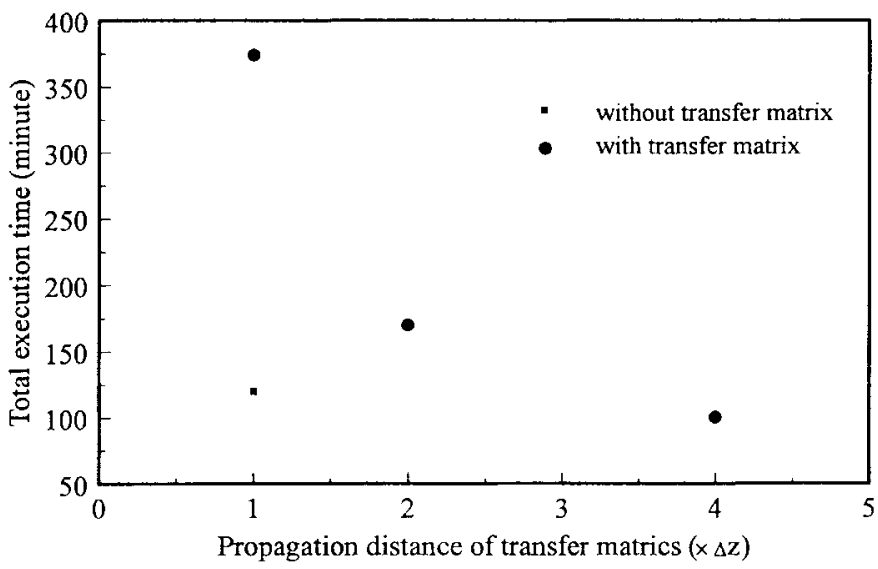

(b)

Fig. 5. Total execution time of (a) 1-D and (b) quasi-3-D time-domain traveling-wave models with different propagation distances.

in Section III-B, the laser is biased at threshold and modulated with a step current. It is noted that the results obtained from the model using a paraxial propagator is almost the same as that using the Padé approximation operator. This is because the transverse light is well confined inside the active layer and spacer layers as well as Bragg reflectors due to the builtin index guiding structure of the laser such that no light is diffracted within the laser cavity.

In order to analyze the influence of light diffraction inside the resonant cavity, the laser structure used in the calculation has to be changed. This can be done by assuming no builtin index-guiding structure inside the spacer layers and Bragg reflectors such that the propagation fields are only transversely guided within the quantum-well active layer in which the diffraction of propagation fields occurs. Furthermore, the core

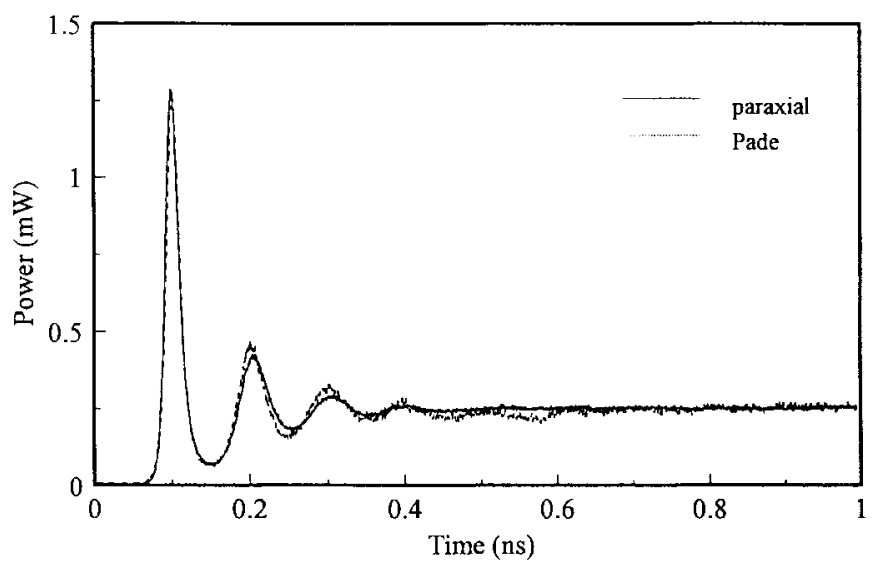

(a)

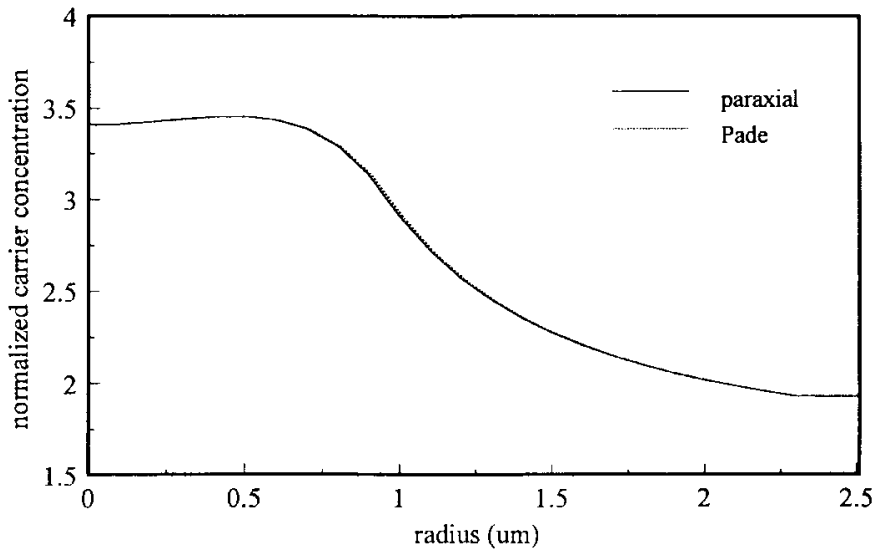

(b)

Fig. 6. (a) The transient response of output power and (b) the steady-state carrier concentration profile of the quasi-3-D time-domain traveling-wave model using a paraxial propagator (solid line) and a Padé $(3,3)$ operator (dotted line)

radius $w$ and the difference of refractive index between the core and cladding regions of the active layer $\Delta n_{a}$ are allowed to change. The corresponding values of the refractive index along the longitudinal direction of the entire multilayer VCSEL structure are listed in Table III.

The influence of light diffraction on the steady-state and dynamic behavior of VCSEL's is demonstrated by equating $w$ and $\Delta n_{a}$ to $0.8 \mu \mathrm{m}$ and 0.4 (i.e., strong index guiding inside the active layer), respectively. Fig. 7(a) and (b) compares the on-axis mode intensities in the longitudinal direction and mode intensities at the QW position in the transverse direction, respectively, from the models using the paraxial propagator and Padé $(3,3)$ operator. It is observed that the optical field is diverges less from the core region of the active 


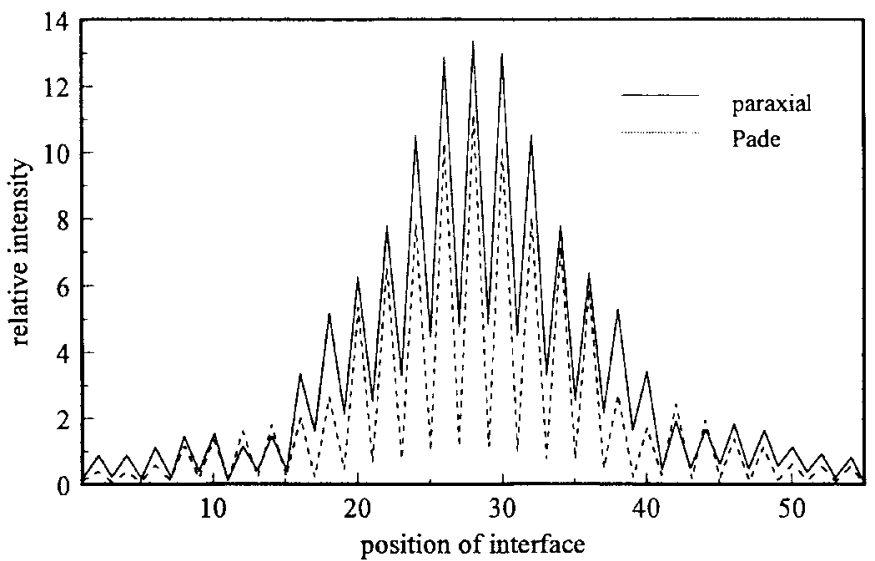

(a)

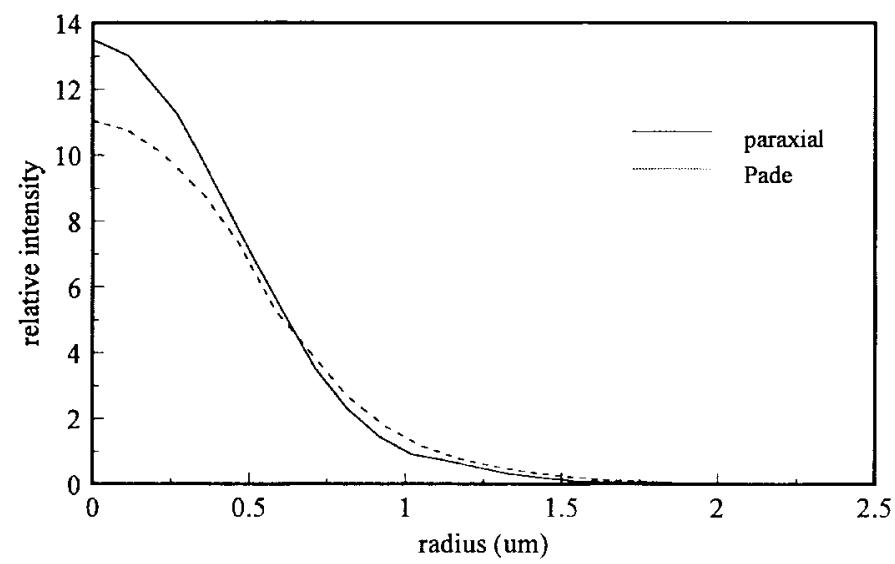

(b)

Fig. 7. (a) On-axis mode intensities in the longitudinal direction and (b) mode intensities at the QW position in the transverse direction calculated from the models using a paraxial propagator and a Padé $(3,3)$ operator. In the calculation, $w$ and $\Delta n_{a}$ are set to 0.8 and $0.4 \mu \mathrm{m}$, respectively.

layer for the case using the paraxial propagator than that using the Padé $(3,3)$ operator. Fig. 8(a) and (b) shows the corresponding dynamic response of the output power and the carrier concentration profile at steady state. As we can see, the output power as well as the relaxation oscillation frequency are overestimated for the case using the paraxial propagator. This is because the degree of light diffraction and total cavity loss are underestimated by the model using the paraxial propagator.

Now, the relative error (i.e., relative to the results obtained from the model using the Padé $(3,3)$ operator) computed from the model using the paraxial propagator as well as Padé operators of orders $(1,1)$ and $(2,2)$ are compared with $w$ and $\Delta n_{a}$ as the variable parameters. It must be noted that it is difficult to evaluate the computational error from the dynamic calculation as the exact solution cannot be obtained. Therefore, the relative error is defined as the average of the relative peak steady-state power of the optical field along the longitudinal direction, that is,

$$
\text { error }=\frac{1}{\text { total no. of } E} \sum_{\forall z} \frac{E(z)-E_{o}(z)}{E_{o}(z)} \times 100 \%
$$

where $E_{o}$ is the steady-state peak power obtained from the model using the Padé $(3,3)$ operator. Fig. 9 shows the influ-

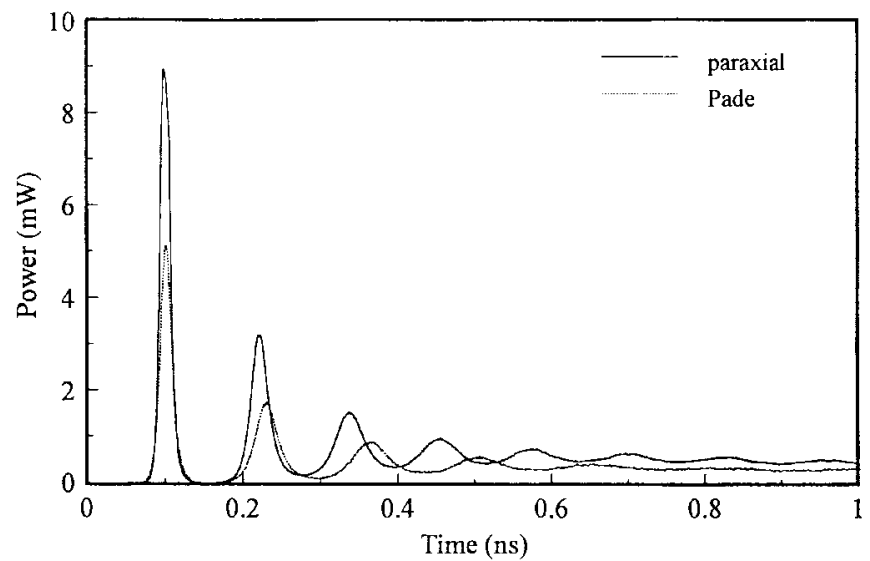

(a)

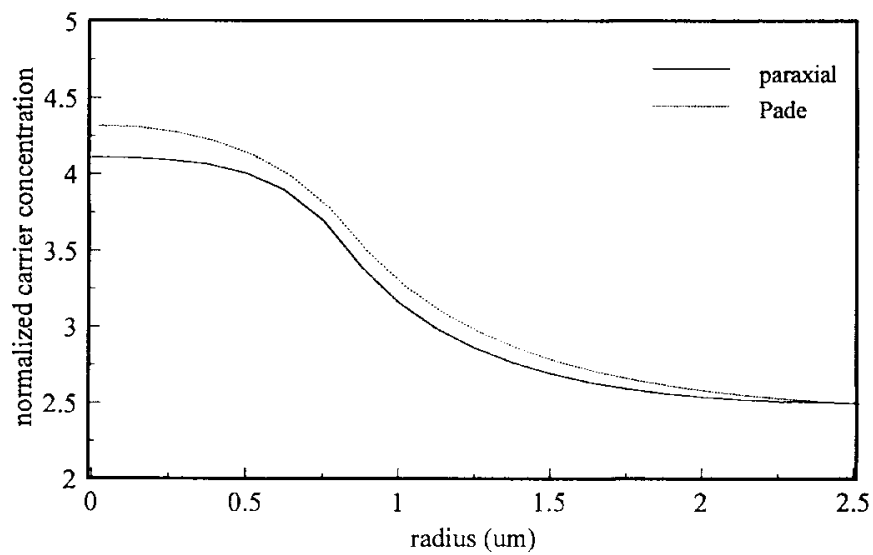

(b)

Fig. 8. The corresponding (a) dynamic response of output power and (b) steady-state carrier concentration distribution calculated form the model using the paraxial propagator (solid line) and the Padé $(3,3)$ operator (dotted line). In the calculation, $w$ and $\Delta n_{a}$ are set to 0.8 and $0.4 \mu \mathrm{m}$, respectively.

ence of $w$ on the relative error of the models with the paraxial propagator and the Padé operators of order $(1,1)$ and $(2,2)$. The value of $w$ is set to $1.0,1.2,1.3,1.5,1.7$, and $1.8 \mu \mathrm{m}$ and the value of $\Delta n_{a}$ is set to 0.6 for all cases. It is observed that the relative error increases with a decrease in $w$ for all the models. This is because the diffraction of light is enhanced for devices with small aperture. In addition, the relative error is minimized for the model using a Padé $(2,2)$ operator. Fig. 10 shows the influence of $\Delta n_{a}$ on the relative error. In the calculation, the value of $\Delta n_{a}$ is set to $0.5,0.55,0.6,0.65$, and 0.7 and the value of $w$ is set to $1.3 \mu \mathrm{m}$ for all models. It is observed that the relative error increases with the increase of $\Delta n_{a}$ but decreases with an increase in $w$. Furthermore, the relative error is minimized for the model using the Padé $(2,2)$ operator. From the above analysis, it is shown that the timedomain model with a Padé operator can be used to analyze the influence of light diffraction in VCSEL's especially for the devices with a strong index-guiding structure and small aperture size.

\section{Discussion AND CONCLUSION}

From the analysis given in Section III, it is shown that the execution time and calculation accuracy of the modified 


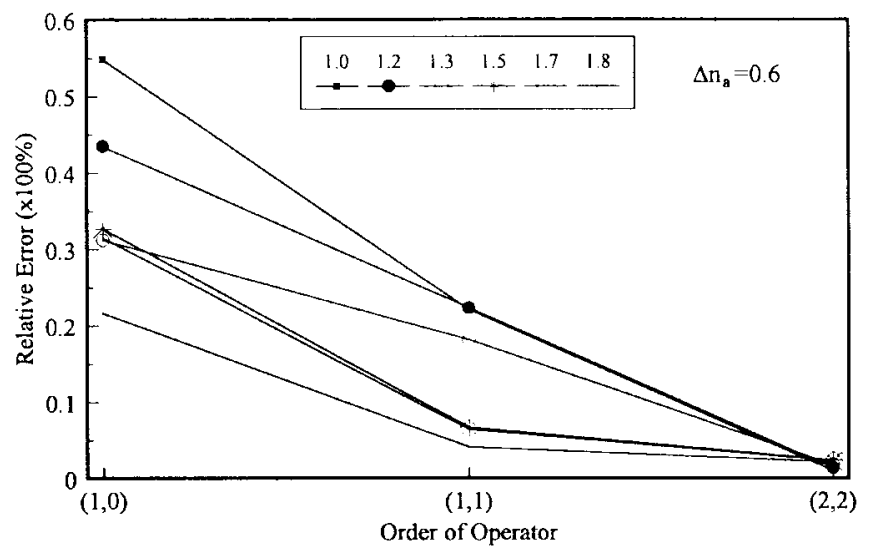

Fig. 9. The relative error against the order of operators used in the models. The $(1,0),(1,1)$, and $(2,2)$ order of operators represent the paraxial propagator and Padé $(1,1)$ and $(2,2)$ operators, respectively. $\Delta n_{a}$ is set to 0.6 and $w$ is varied from 1.0 to $1.8 \mu \mathrm{m}$.

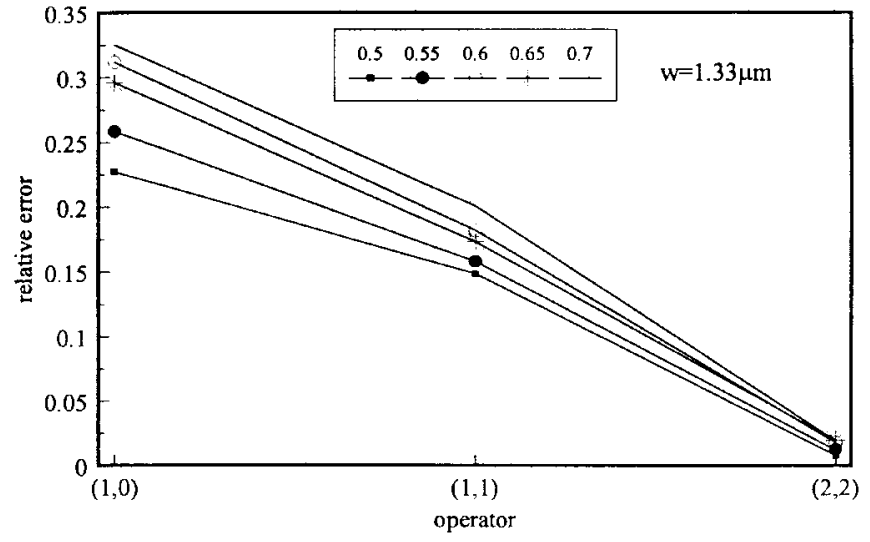

Fig. 10. The relative error against the order of operators used in the models The $(1,0),(1,1)$, and $(2,2)$ order of operators represents the paraxial propagator and Padé $(1,1)$ and $(2,2)$ operators, respectively. $w$ is set to $0.8 \mu \mathrm{m}$ and $\Delta n_{a}$ is varied from 0.5 to 0.7 .

traveling-wave model has been improved by using the timedependent transfer matrix and wide-angle beam propagation method. The performance of the laser models can be summarized as follows.

- The execution time of the 1-D model can be reduced by more than a factor of two by using the transfer matrix due to the high efficiency in the calculation of (9). Therefore, the 1-D model helps enormously in shortening the design cycle of VCSEL's. For examples, the 1$\mathrm{D}$ model is suitable for the analysis of the transient response of coupled-cavity VCSEL's [15] or the design of VCSEL's for self-sustained pulsation operation [16]. This is because in these device structures light diffraction is not a dominant factor.

- The total execution time of the quasi-3-D model has less improvement than the 1-D model. This is because of the low efficiency in the calculation of (9) when inverse operation of a matrix is involved in the calculation.

- The calculation accuracy of the quasi-3-D model has been improved significantly by using a Padé operator. This improvement of the traveling-wave model is well suited for the analysis of VCSEL's with a strong index-guiding structure and small aperture size such as those with an index-guided structure [12] or double-oxide-confined structure [17]. This is because the off-axis diffraction of light inside and at the interface of the dielectric layers can be accurately taken into consideration.

- It is noted that the quasi-3-D model using the Padé (3, 3) operator takes approximately $6 \mathrm{~h}$ to simulate the 1 ns dynamic response of VCSEL's. In fact, the coding of this simulation program has not been optimized and it is believed that the total execution time can be easily reduced by at least half. To the best of our knowledge, this model is the fastest and the simplest that we can find in the literature. In addition, the calculation accuracy is sufficient to estimate most of the important characteristics of VCSEL's such as diffraction loss, self-focusing effects, and spatial hole burning of carrier concentration.

In the above analysis, the self-heating effects are ignored in the calculation. This is because the phenomena of off-axis light diffraction has been the main concern of this paper. In fact, it is possible to include a thermal heat equation into the model to analyze the influence of self-heating effects on the modulation response of VCSEL's, and this will form the subject of our future study. In conclusion, the execution time and calculation accuracy of the time-domain traveling-wave model of VCSEL's are improved by using the transfer-matrix and wide-angle beam propagation methods. It is shown that the total execution time of the 1-D time-domain traveling-wave model can be reduced by half when using the transfer matrix. However, the implementation of the transfer matrix in a 3-D model requires the calculation of inverse operation of matrices such that the total execution time of the 3-D model may not be reduced as much as the 1-D model. On the other hand, the influence of light diffraction on the dynamic behavior of VCSEL's has been carefully considered through the use of a Padé operator in the calculation. It is shown in the calculation that diffraction of light is important for devices with a strong index-guiding structure, $\Delta n_{a} \geq 0.5$, and small aperture size, $w \leq 1.3 \mu \mathrm{m}$. However, the model using the Padé $(3,3)$ operator is sufficient for eliminating error due to the influence of diffraction loss.

\section{APPENDIX A}

The effective change of permittivity $\Delta \varepsilon$ of the quasi-3-D waveguide geometry is defined as [9]

$$
\begin{aligned}
& \Delta \varepsilon(r, z) \\
& =n_{g} \times \begin{cases}\left(2 \Delta n_{b}(r, z)+\Delta n(N)\right)-i\left(g(N)-\alpha_{s}\right) / k_{o}, \\
\text { active layer } & \text { (A1) } \\
2 \Delta n_{b}(r, z)+i \alpha_{s} / k_{o}, & \text { elsewhere }\end{cases}
\end{aligned}
$$

where $\Delta n_{b}(r, z)$ is the difference of the built-in refractive index relative to the core region and $\alpha_{s}$ is the absorption and scattering loss inside the core region and the cladding regions. $\Delta n$ is the carrier-induced index change and is given by

$$
\Delta n(N)=b_{N} \cdot \log \left(N / N_{1}\right)
$$

where $b_{N}$ and $N_{1}$ are some fitted parameters. The effective change of permittivity $\Delta \varepsilon_{\text {eff }}$ of the 1-D waveguide geometry 
is given by [8]

$$
\begin{aligned}
& \Delta \varepsilon_{\mathrm{eff}}(z) \\
& \quad=\int_{0}^{\infty} \Delta \varepsilon(r, z) E(r, z) r d r d \theta / \int_{0}^{\infty} E(r, z) r d r d \theta .
\end{aligned}
$$

In (A1)-(A3), it is noted that the influence of spatial hole burning on the propagation fields is also taken into consideration through carrier-induced refractive index change.

\section{APPENDIX B}

The complete set of plane waves describing the reflected and transmitted fields $\underline{\Psi}_{r}$ and $\underline{\Psi}_{t}$ along the dielectric interface is given by [18]

$$
\begin{aligned}
& \underline{\Psi}_{r}(r)=\int_{0}^{\infty} k_{t} r_{21 p}\left(k_{t}\right) \underline{\hat{\Psi}}_{i}\left(k_{t}\right) J_{0}\left(k_{t} r\right) d k_{t} \\
& \underline{\Psi}_{t}(r)=\int_{0}^{\infty} k_{t} t_{21 p}\left(k_{t}\right) \underline{\hat{\Psi}}_{i}\left(k_{t}\right) J_{0}\left(k_{t} r\right) d k_{t}
\end{aligned}
$$

where $k_{t}$ is the wavevector in the transverse direction and $J_{0}$ is the Bessel function of the first kind of zeroth order. $r_{21 p}$ and $t_{21 p}$ are the plane wave reflection and transmission coefficients, respectively. $\underline{\underline{\Psi}}_{i}$ is the Fourier-Bessel transform of the incident electrical field $\underline{\Psi}_{i}$ and is defined as

$$
\underline{\hat{\Psi}}_{i}\left(k_{t}\right)=\int_{0}^{\infty} r \underline{\Psi}_{i}(r) J_{0}\left(k_{t} r\right) d r
$$

If only the fundamental mode is assumed to be supported by the laser cavity, the reflected and transmitted fields given in (B1)-(B3) can be discretized and written in a matrix formation as shown below:

$$
\begin{aligned}
& \underline{\Psi}_{r}(r)=\left[B_{0}\right]^{-1}\left[R_{21 p}\right]\left[B_{0}\right] \underline{\Psi}_{i}(r) \\
& \underline{\Psi}_{t}(r)=\left[B_{0}\right]^{-1}\left[T_{21 p}\right]\left[B_{0}\right] \underline{\Psi}_{i}(r)
\end{aligned}
$$

where $\left[B_{0}\right]$ and $\left[B_{0}\right]^{-1}$ are the Bessel function operators. It can be shown that the elements of $\left[B_{0}\right]$ and $\left[B_{0}\right]^{-1}, b_{m, n}$ and $\tilde{b}_{m, n}$, can be written as [18]

$$
\begin{aligned}
& b_{m, n}=\frac{1}{W_{t}^{2}} \frac{J_{0}\left(p_{m} p_{n} / p_{M+1}\right)}{J_{1}^{2}\left(p_{m}\right)} \\
& \tilde{b}_{m, n}=\frac{2}{K^{2}} \frac{J_{0}\left(p_{m} p_{n} / p_{M+1}\right)}{J_{1}^{2}\left(p_{n}\right)}
\end{aligned}
$$

where $m$ and $n(=1, \cdots, M)$ are some integers, $M$ is the total number of grid points along the transverse direction, $W_{t}$ is the radius of the laser cavity, $p_{m}$ is the $m$ th zero of $J_{0}$ (i.e., $\left.J_{0}\left(p_{m}\right)=0\right)$ and $K=p_{M+1} / W_{t}$. Hence, $\left[R_{21}\right]$ and $\left[T_{21}\right]$ given in (6) and (15) can be written as

$$
\begin{aligned}
{\left[R_{21}\right] } & =\left[B_{0}\right]^{-1}\left[R_{21 p}\right]\left[B_{0}\right] \\
{\left[T_{21}\right] } & =\left[B_{0}\right]^{-1}\left[T_{21 p}\right]\left[B_{0}\right] .
\end{aligned}
$$

The reflection and transmission matrices $\left[R_{21 p}\right]$ and $\left[T_{21 p}\right]$ at the dielectric interface are defined as

$$
\left[R_{21 p}\right]=\left[\begin{array}{ccc}
r_{21 p}\left(k_{t 1}\right) & & 0 \\
0 & & r_{21 p}\left(k_{t M}\right)
\end{array}\right]
$$

$$
\left[T_{21 p}\right]=\left[\begin{array}{ccc}
t_{21 p}\left(k_{t 1}\right) & & 0 \\
0 & & t_{21 p}\left(k_{t M}\right)
\end{array}\right]
$$

where $k_{t m}=p_{m} / W_{t}$ for $m=1, \cdots, M$. If the dielectric interface are assumed to be polarization-independent, the elements of the reflection and transmission matrices of the incident transverse fields $r_{21 p}$ and $t_{21 p}$ can be expressed as

$$
\begin{aligned}
& r_{21 p}\left(k_{t m}\right)=\frac{\beta_{2}-\beta_{1}}{\beta_{1}+\beta_{2}} \\
& t_{21 p}\left(k_{t m}\right)=\frac{2 \beta_{2}}{\beta_{1}+\beta_{2}}
\end{aligned}
$$

where $\beta_{1}=\sqrt{n_{1}^{2} k_{o}^{2}-k_{t m}^{2}}$ and $\beta_{2}=\sqrt{n_{2}^{2} k_{o}^{2}-k_{t m}^{2}}$. Using this approach, the effects of off-axis light propagation at the interface of the dielectric layers can also be taken into consideration.

If normal incident is assumed in calculation of the 1-D model (i.e., $k_{t m}=0$ ), $\left[R_{21}\right]$ and $\left[T_{21}\right]$ can be simplified to

$$
\begin{aligned}
{\left[R_{21}\right] } & =\frac{n_{2}-n_{1}}{n_{2}+n_{1}}[I] \\
{\left[T_{21}\right] } & =\frac{2 n_{2}}{n_{2}+n_{1}}[I]
\end{aligned}
$$

where $[I]$ has the dimensions $M \times M$.

\section{ACKNOWLEDGMENT}

The author would like to thank P. Kan and S. K. Que of the Department of Electrical and Electronic Engineering, Hong Kong University, for their computational work and the reviewer for his/her constructive comments.

\section{REFERENCES}

[1] D. Tauber, G. Wang, R. S. Geels, J. E. Bowers, and L. A. Coldern, "Large and small signal dynamics of vertical cavity surface emitting lasers," Appl. Phys. Lett., vol. 62, pp. 325-327, 1993.

[2] G. Shtengel, H. Temkin, P. Brusenbach, T. Uchida, M. Kim, C. Parsons, W. E. Quinn, and S. E. Swirhun, "High speed vertical cavity surface emitting laser," IEEE Photon. Technol. Lett., vol. 5, pp. 1359-1362, 1993.

[3] A. Valle, J. Sarma, and K. A. Shore, "Spatial hole burning effects on the dynamics of vertical cavity surface emitting lasers," IEEE J. Quantum Electron., vol. 31, pp. 1423-1431, 1995.

[4] J. Y. Law and G. P. Agrawal, "Effects of spatial hole burning on gain switching in VCSEL's," IEEE J. Quantum Electron., vol. 33, pp. 462-468, Mar. 1997.

[5] R. R. Burton, M. S. Stern, P. C. Kendall, and P. N. Robson, "Modeling of diffraction in pillar vertical cavity surface emitting lasers with embedded Bragg layers," Opt. Quantum Electron., vol. 28, pp. 1677-1684, 1996.

[6] Y. G. Zhao and J. G. McInerney, "Transverse-mode control of vertical cavity surface emitting lasers," IEEE J. Quantum Electron., vol. 32, pp. 1950-1958, Nov. 1996.

[7] S. F. Yu, "Analysis and design of vertical cavity surface emitting lasers for self-sustained pulsation operation," IEEE J. Quantum Electron., vol. 34, pp. 497-505, Mar. 1998.

[8] _ _Dynamic behavior of vertical cavity surface emitting lasers," IEEE J. Quantum Electron., vol. 32, no. 7, pp. 1168-1179, 1996.

[9] S. F. Yu and C. W. Lo, "Influence of transverse modes on the dynamic response of vertical cavity surface emitting lasers," Proc. Inst. Elect. Eng., vol. 143, pt. J, no. 3, pp. 189-194, 1996.

[10] L. M. Zhang, S. F. Yu, M. C. Nowell, D. D. Marcenac, J. E. Carroll, and R. G. S. Plumb, "Dynamic analysis of radiation and side mode suppression in second order DFB lasers using time-domain largesignal traveling wave model," IEEE J. Quantum Electron., vol. 30, pp. 1389-1395, June 1994. 
[11] M. G. Davis and R. F. O'Dowd, "A transfer matrix method based large signal dynamic model for multielectrode DFB lasers," IEEE J. Quantum Electron., vol. 30, pp. 2458-2466, Nov. 1994.

[12] Y. Kohama, Y. Ohiso, K. Tateno, and T. Kurokawa, "0.85 $\mu \mathrm{m}$ vertical cavity surface emitting laser diode arrays grown on p-type GaAs substrate," IEEE Photon. Technol. Lett., vol. 9, pp. 280-282, Mar. 1997.

[13] G. R. Hadley, "Wide-angle beam propagation using Padé approximant operators," Opt. Lett., vol. 17, pp. 1426-1428, 1991.

[14] N. K. Dutta, "Analysis of current spreading, carrier diffusion, and transverse mode guiding in surface emitting lasers," J. Appl. Phys., vol. 68, pp. 1961-1963, 1990.

[15] P. Pellandini, R. P. Stanley, R. Houdre, U. Oesterie, M. Llegems, and C. Weisbuch, "Dual wavelength laser emission from a coupled semiconductor microcavity," Appl. Phys. Lett., vol. 71, pp. 864-866, 1997.
[16] D. G. H. Nugent, R. G. S. Plumb, M. A. Fisher, and D. A. O. Davies, "Self-pulsation in vertical cavity surface emitting lasers," Electron. Lett., vol. 31 , no. 1 , pp. 43-44, 1995

[17] T. H. Oh, D. L. Huffaker, and D. G. Deppe, "Comparison of vertical cavity surface emitting lasers with half-wave cavity spacer confined by single or double oxide apertures," IEEE Photon. Technol. Lett., vol. 9, pp. 875-877, 1997.

[18] J. F. P. Seurin and S. L. Chuang, "Discrete bessel transform and beam propagation method for modeling of vertical cavity surface emitting lasers," J. Appl. Phys., vol. 82, no. 5, pp. 2007-2016, 1997.

S. F. Yu, for photograph and biography, see p. 505 of the March 1998 issue of this JOURNAL. 